\title{
Aprendizado e experiência na formação profissional em enfermagem obstétrica acerca dos desfechos relacionados à prematuridade e baixo peso ao nascer
}

\author{
Learning and experience in professional training in obstetric nursing about the outcomes \\ related to prematurity and low birth weight
}
Aprendizaje y experiencia en la formación profesional en enfermería obstétrica sobre los resultados relacionados con la prematuridad y el bajo peso al nacer

Bruna de Oliveira Moraes ${ }^{1 *}$, Sineide Santos de Souza ${ }^{1}$, Anny Beatriz Costa Antony de Andrade ${ }^{2}$, Aimée de Queiroz Carvalho ${ }^{1}$, Alessandra Pinheiro Vidal ${ }^{1}$, Rizioléia Marina Pinheiro Pina ${ }^{1}$.

\begin{abstract}
RESUMO
Objetivo: Apresentar a experiência vivenciada na residência do Programa de Enfermagem Obstétrica de uma universidade pública do estado do Amazonas durante atividades práticas desenvolvidas em maternidades públicas em um município do Amazonas, relacionada aos nascimentos de bebês prematuros e de baixo peso. Relato de experiência: Trata-se de um estudo descritivo, tipo relato de experiência sobre a vivência de residentes em enfermagem obstétrica na assistência as parturientes com bebês prematuros e com baixo peso ao nascer. A experiência vivenciada na formação permitiu a observação e detecção de fatores de risco que influenciam de forma direta e/ou indireta no desfecho de prematuridade e baixo peso ao nascer, como gravidez na adolescência, inadequada assistência pré-natal, ou ainda a ausência desta, baixo nível socioeconômico, presença de comorbidades. Os desfechos observados exercem grande impacto na saúde da população em estudo, colaborando no aumento da morbimortalidade materno-infantil. Considerações finais: A residência em enfermagem obstétrica proporciona o aperfeiçoamento de técnicas e habilidades, bem como obtenção e consolidação de novos conhecimentos, tais ações podem estar diretamente ligadas ao formato de ensino que a residência oportuniza, o que resulta em prestação de assistência especializada e baseada em evidências científicas à população materno-infantil.
\end{abstract}

Palavras-chave: Recém-nascido prematuro, Recém-nascido de baixo peso, Cuidado pré-natal.

\begin{abstract}
Objective: To present the experience lived at the residence of the Obstetric Nursing Program of a public university in the state of Amazonas during practical activities developed in public maternity hospitals in a municipality in Amazonas, related to the births of premature and low weight babies. Experience report: This is a descriptive study, an experience report about the experience of residents in obstetric nursing in assisting parturients with premature babies and low birth weight. The experience in the training allowed the observation and detection of risk factors that directly and / or indirectly influence the outcome of prematurity and low birth weight, such as teenage pregnancy, inadequate prenatal care, or even the absence of this, low socioeconomic level, presence of comorbidities. The observed outcomes have a great impact on the health of the study population, helping to increase maternal and child morbidity and mortality. Final considerations: The residency in obstetric nursing provides the improvement of techniques and skills, as well as obtaining and consolidating new knowledge, such actions may be directly linked to the teaching format that the residency provides, which results in the provision of specialized assistance and based on scientific evidence to the maternal and child population.
\end{abstract}

Keywords: Premature newborn, Low weight newborn, Prenatal care.

1Universidade Federal do Amazonas (UFAM), Manaus - AM. *E-mail: moraesbruna192070@gmail.com ${ }^{2}$ Instituto Leônidas \& Maria Deane (ILMD/FIOCRUZ Amazônia), Manaus - AM.

SUBMETIDO EM: 10/2020

ACEITO EM: 11/2020

PUBLICADO EM: 2/2021 


\section{RESUMEN}

Objetivo: Presentar la experiencia vivida en la residencia del Programa de Enfermería Obstétrica de una universidad pública del estado de Amazonas durante las actividades prácticas desarrolladas en maternidades públicas de un municipio de Amazonas, relacionadas con los nacimientos de bebés prematuros y de bajo peso. Informe de experiencia: Se trata de un estudio descriptivo, relato de experiencia sobre la experiencia de residentes de enfermería obstétrica en la asistencia a parturientas con prematuros y bajo peso al nacer. La experiencia en la formación permitió la observación y detección de factores de riesgo que influyen directa y / o indirectamente en el desenlace de la prematuridad y el bajo peso al nacer, como el embarazo adolescente, la atención prenatal inadecuada, o incluso la ausencia de esta, nivel socioeconómico bajo, presencia de comorbilidades. Los resultados observados tienen un gran impacto en la salud de la población de estudio, ayudando a incrementar la morbilidad y mortalidad materna e infantil. Consideraciones finales: La residencia en enfermería obstétrica proporciona el perfeccionamiento de técnicas y habilidades, así como la obtención y consolidación de nuevos conocimientos, acciones que pueden estar directamente ligadas al formato docente que brinda la residencia, lo que se traduce en la prestación de asistencia especializada y basada en evidencia científica a la población materna e infantil.

Palabras clave: Recién nacido prematuro, Recién nacido de bajo peso, Atención prenatal.

\section{INTRODUÇÃO}

As residências multiprofissionais e uniprofissionais em saúde são programas de ensino consolidados pelo Ministério da Educação (MEC) e reconhecido como pós-graduação lato sensu. O programa de residência manifesta-se como um novo modelo de prestação dos serviços públicos fundamentado nos princípios do Sistema Único de Saúde (SUS) (SILVA LB e CAPAZ R, 2013; SILVA CA e DALBELLO-ARAUJO M, 2019).

A Residência em Enfermagem Obstétrica é tida como grande oportunidade de aprendizado e aperfeiçoamento profissional, permitindo a qualificação do residente dentro dos serviços de saúde voltados para a assistência à saúde da mulher e neonatal, promovendo ensino e formação, com preceptores nos campos de práticas. Este modelo de ensino, tem ganhado grande destaque no meio acadêmico, em virtude de sua qualificação diferenciada, apontado como padrão ouro de especialização, com destaque ainda para a contribuição substancial nos serviços e saúde da população atendida (SILVA CA e DALBELLO-ARAUJO M, 2019).

$\mathrm{Na}$ assistência a mulher e ao neonato, observa-se que a prematuridade e o baixo peso ao nascer (BPN) estão entre os fatores determinantes quando se trata de mortalidade neonatal, estando o baixo peso associado tanto à prematuridade, quanto ao crescimento intrauterino restrito (CIUR) (GONZAGA ICA, et al., 2016; MOREIRA Al, et al., 2018). Define-se como CIUR a deficiência mórbida do feto em alcançar sua capacidade de crescimento intrauterino, constituindo por sua vez grave motivo de morbimortalidade perinatal (BRASIL, 2012).

A Organização Mundial da Saúde (OMS) define como prematuro, o nascido vivo com menos de 37 semanas completas, independente do peso (GUIMARÃES EAA, et al., 2017). O BPN é definido como peso inferior a $2.500 \mathrm{~g}$ ao nascimento, não interferindo o período gestacional, este por sua vez representa notável problema de saúde, com altas taxas de morbimortalidade neonatal (BRASIL, 2013). O BPN é um importante preditivo de desfechos desfavoráveis quando nos referimos à saúde infantil, dentre os quais estão o atraso no crescimento e desenvolvimento, as infecções que acometem o trato respiratório e gastrointestinal e ainda de maneira mais crítica a mortalidade infantil (ARNOLD L, et al., 2016). Presume-se que 15 a 20\% dos recémnascidos em todo o mundo apresentem BPN, o que representaria mais de 20 milhões de nascimentos por ano (WHO, 2014).

Silva PNS, et al. (2018) e Moreira Al, et al. (2018) referem que o nascimento prematuro e BPN representam graves problemas de saúde, estando entre as maiores causas de mortalidade infantil e sua ocorrência está associada à inúmeros fatores de risco. Entre os fatores de risco há características socioeconômicas como a idade materna, características individuais das gestantes, o uso de drogas lícitas e ilícitas, características obstétricas, infecções durante o período gestacional, características fetais e relacionadas à inadequada assistência pré-natal. O cuidado pré-natal é essencial para a promoção de um nascimento saudável, sendo 
fundamental para a redução da morbimortalidade materna e fetal (BARRETO CTG, et al., 2019). A OMS preconiza que sejam realizadas ao menos oito consultas de pré-natal, a fim de assegurar às mães a redução dos efeitos adversos de uma assistência pré-natal inadequada, bem como a redução da mortalidade perinatal e melhoria na experiência das mulheres com os cuidados ofertados nesse componente (WHO, 2016).

Melo EC, et al. (2015), salientam que quando o cuidado pré-natal é realizado de maneira adequada, é um elemento eficaz para a prevenção da morbimortalidade materna e infantil, uma vez que coopera para desfechos mais oportunos baseados na realização de procedimentos considerados básicos, como a realização de exames clínicos-laboratoriais, assim como acompanhamento da gestação por meio das consultas agendadas periodicamente, o que permite a detecção precoce de complicações e o tratamento de forma oportuna, atenuando os fatores de risco que apresentam problemas para o bem-estar do binômio.

\section{RELATO DE EXPERIÊNCIA}

Trata-se de um relato de experiência sobre a vivência de uma enfermeira residente do Programa de Enfermagem Obstétrica de uma universidade pública do estado do Amazonas durante atividades práticas desenvolvidas em maternidades públicas em um município do Amazonas.

A carga horária do programa de residência é de 5.760 (cinco mil setecentos e setenta) horas, dispostas em 4.608 (quatro mil seiscentos e oito) horas destinadas a estratégias práticas e 1.152 (mil cento e cinquenta e duas) horas voltadas a estratégias teóricas ou teórico-práticas, com regime de 60 (sessenta) horas semanais, nas quais ocorre a assistência de enfermagem em unidades de saúde da Atenção Básica (AB) e hospitalar à saúde da mulher e família, bem como durante o seu ciclo gravídico-puerperal.

Durante a assistência de enfermagem desenvolvida nas maternidades, observou-se frequência significativa de mulheres em trabalho de parto prematuro, o nascimento de crianças pré-termo e de crianças com BPN. A observação desse cenário causou inquietações na profissional residente, levando-a a identificar os possíveis fatores de risco associados aos desfechos de prematuridade e BPN, em gestantes que buscaram atendimento em maternidades públicas do município de Manaus.

Quando a paciente adentra a unidade de saúde, faz-se então sua anamnese e exame físico, bem como avaliação dos registros contidos nas cadernetas da gestante, ultrassonografias obstétricas, exames realizados durante o pré-natal e na maternidade, além de informações contidas nos prontuários, tais ações se aplicam a todas as mulheres. Uma vez identificada a internação de gestantes com diagnóstico de trabalho de parto prematuro, ou com idade gestacional sugestiva de prematuridade, passava-se a investigar de forma mais detalhada a história pregressa e histórico da gestação atual dessas parturientes durante a assistência prestada, a fim de compreender e buscar quais potenciais fatores de risco apresentados poderiam ter contribuído para os desfechos.

Observou-se um número significativo de mulheres na faixa etária considerada entre 12 a 18 anos, em menor frequência mulheres com 35 anos ou mais. Quanto às demais características sociodemográficas, as pacientes apresentavam baixa escolaridade, baixo nível socioeconômico e a maioria solteira. Identificou-se apenas uma parturiente usuária de álcool e drogas ilícitas. Em relação às características obstétricas, as intercorrências comumente apresentadas por mulheres com 35 anos ou mais e que tiveram como desfecho o parto prematuro e BPN, foram síndromes hipertensivas e rotura prematura de membranas ovulares (RPMO).

Notou-se na maioria das cadernetas das gestantes o registro de apenas cinco consultas, algumas mulheres não realizaram o pré-natal. Identificou-se a ausência de registros de exames de rotina preconizados no período gestacional na caderneta. A partir desse achado buscou-se orientar e sensibilizar os profissionais da atenção básica, quanto ao preenchimento adequado da caderneta da gestante, haja vista ser uma importante ferramenta para a identificação de forma precoce de possíveis alterações, que necessitam de intervenções a fim de prevenir desfechos inesperados como a prematuridade e o baixo peso ao nascer. Orientaram-se também os residentes de enfermagem obstétrica do primeiro ano de formação, já que destinam boa parte do aprendizado na atenção básica, com atividades diretas na assistência pré-natal, com vistas a favorecer um olhar mais atencioso para as questões supracitadas. 


\section{DISCUSSÃO}

No decorrer das práticas na residência foi possível verificar que é frequente a presença de adolescentes grávidas nas consultas de pré-natal, em sua maioria, relacionadas à primeira gestação, mas também havia multigestas. É aparente pela linguagem corporal, as expressões de medo, ansiedade e inquietações que elas refletem, muitas vezes por não compreenderem a complexidade de se tornar mãe e assumir inúmeras responsabilidades.

Ao assistir a adolescente, é necessário que os profissionais de saúde desenvolvam sensibilidade e empatia, que estejam despidos de preconceitos e/ou pré-julgamentos, usem linguagem acessível com vistas a compreensão das orientações realizadas, reforcem a importância do comparecimento às consultas prénatais e a coparticipação com sua saúde e a do bebê, bem como promover o vínculo com a gestante, familiares e comunidade, de modo a construir uma rede de apoio. O apoio advindo da família colabora para o desenvolvimento de uma gravidez tranquila e fortalece as adolescentes, tendo potencial de amenizar impactos que possam afetar de maneira negativa a saúde, sendo fundamental para a segurança nessa etapa da maternidade (BRAGA IF, et al., 2014).

A gravidez na adolescência está associada ao parto prematuro e BPN, Viellas EF, et al. (2012) identificaram em estudo, que a gravidez na adolescência está associada à desfechos negativos no recémnascido e que as adolescentes grávidas estão entre as classes mais pobres, de baixa escolaridade e têm menor atenção na assistência pré-natal, estando seus filhos vulneráveis aos desfechos acima mencionados.

Esse achado mostra concordância com a experiência vivenciada na Unidade Básica de Saúde (UBS), onde observou-se que as adolescentes eram as mais faltosas nas consultas de pré-natal, e quando presentes, a linguagem corporal demonstrava que não atentavam para as informações passadas pela equipe de saúde, sendo a maioria de baixo nível socioeconômico e com ensino médio incompleto.

Por outro lado, constatou-se que o estabelecimento de uma relação de confiança é essencial para que a adolescente gestante participe do pré-natal, seja envolvida nas atividades disponíveis na UBS e para a aproximação da família no momento único, especial e cheio de dúvidas e incertezas que é a gravidez. Assim, acredita-se que o desenvolvimento de estratégias no formato educação em saúde com metodologias voltadas para o público adolescente em escolas e nas UBS possam fomentar informações que contribuam, ainda que indiretamente, para a redução das taxas de gravidez na adolescência não desejada, além de ampliar a captação precoce das adolescentes para o início do pré-natal, para o acompanhamento efetivo, e por consequência, minimizar os desfechos de prematuridade e de baixo peso em recém-nascidos nessa população.

De acordo com estudo realizado por Gonzaga ICA, et al. (2016), a inadequada assistência pré-natal está dentre as causas principais que levam ao BPN e a prematuridade, dado que, uma prestação de serviço de saúde realizada ao nível de atenção básica possibilita o diagnóstico precoce, o tratamento de possíveis complicações que podem ocorrer durante o período gestacional, sendo factível a redução ou até mesmo a eliminação de condições de riscos, propiciando assim, um desfecho satisfatório para a mãe e o feto.

Considera-se que a inadequada assistência pré-natal é importante fator de risco para nascimentos prétermos e de BPN, haja vista a notória relevância na prevenção e deteç̧ão precoce de possíveis comorbidades que possam provocar efeitos indesejáveis para o binômio, como a Hipertensão, Diabetes Gestacional, CIUR, ganho ponderal inadequado da gestante, presença de infecções, como Infecção do Trato Urinário (ITU); Sífilis; Toxoplasmose; Hepatites, entre outras, e intervenções são realizadas no sentido de evitar complicações e desfechos negativos. É sabido que a ITU pode desencadear o Trabalho de Parto Prematuro (TPP) e gerar complicações para o crescimento e desenvolvimento fetal, assim como a sífilis. Gois ALC (2010) refere que a gestação quando associada a ITU, acarreta mal prognóstico, tendo como principais complicações o TPP e o Parto Prematuro.

E com base na identificação de alguns desses diagnósticos, toma-se, portanto, as devidas precauções, bem como à realização de encaminhamentos ao pré-natal de Alto Risco, uma vez que essa gestante necessita de atenção ainda mais especializada, ressaltando para ela a importância de se manter presente também nas consultas na UBS. 
Mediante a experiência e aprendizado previamente adquiridos durante a formação acadêmica e no Programa de Residência por meio das atividades práticas desenvolvidas na $A B$, como nas consultas de prénatal, é clara a relevância do acompanhamento adequado nesse nível de atenção, sendo essencial na identificação antecipada de eventos que possam ser prejudiciais para o curso da gestação, como também intervir em tempo oportuno, por meios de ações de saúde, na ocorrência dos desfechos de prematuridade e BPN. Observou-se ainda que as parturientes, em sua maioria, eram primíparas e que a gestação não foi planejada. Oliveira AA, et al. (2019) destacam que a gravidez não planejada está associada ao hábito de fumar durante a gestação, uso inadequado ou não adesão do uso de ácido fólico e início tardio do pré-natal.

De encontro aos resultados deste estudo, verificou-se por meio da anamnese e informações contidas no cartão da gestante, que as mulheres engravidaram sem nenhum planejamento, não faziam uso de métodos contraceptivos, era a primeira gestação e algumas não haviam feito uso do ácido fólico e sulfato ferroso conforme preconizado durante o período gestacional.

Os achados corroboram com o mencionado pela literatura acerca do assunto, a qual indica que as variáveis socioeconômicas, entre elas, a baixa escolaridade, pertencer a classes econômicas menos favorecidas, ser solteira, ausência de planejamento da gravidez, a história reprodutiva, a presença de intercorrências durante o período gestacional, o uso de álcool ou drogas ilícitas durante a gestação, a gravidez na adolescência, a inadequação ou não adesão à assistência pré-natal, a dificuldade de acesso aos serviços de saúde e o parto pré-termo anterior estão entre os fatores de risco mais comuns associados diretamente aos desfechos de prematuridade e BPN. Entretanto, os autores ressaltam o importante e determinante papel da assistência pré-natal para desfechos positivos relacionados ao binômio (GONZAGA ICA, et al., 2016; GUIMARÃES EAA, et al., 2017; SILVA PNS, et al., 2018; OLIVEIRA AA, et al., 2019).

Estudo realizado na Amazônia Legal, em uma maternidade referência no Pará demonstrou fatores de maior risco para o BPN na população estudada: idade gestacional, em especial de 22 a 36 semanas, evidenciando a prematuridade como causa principal de BPN, fatores socioeconômicos como baixo nível de escolaridade materna e baixa renda, idade materna igual ou maior que 35 anos, doenças como ITU, hipertensão materna, rotura prematura de membranas e vulvo vaginite, além de internação na gestação (CHERMONT A, et al., 2019).

Alguns bebês com BPN e prematuridade necessitaram de assistência especializada como recursos humanos e equipamentos de alta tecnologia para a manutenção da vida, e apesar do suporte adequado, alguns chegaram ao desfecho do óbito. A realidade vivenciada corrobora com o estudo de Figueiró-Filho EA, et al. (2014), o qual evidenciou que os bebês prematuros e de BPN possuem maior risco de adoecer e morrer, de infecções e complicações devido o desenvolvimento fetal incompleto, principalmente no período neonatal. Além de comprometimento neurológico, oftalmológico, pulmonar, e outras comorbidades que podem causar impacto no crescimento e desenvolvimento, bem como na dinâmica familiar por demandar um nível de cuidado complexo. Para Teixeira AG, et al. (2018), o cuidado poderá ser necessário ao longo da vida, provocando um custo socioeconômico elevado para o país.

A experiência gerou um alerta iminente da residente para a população considerada grupo de risco para os desfechos abordados nesse relato, pois expressam grande problema de saúde pública, em virtude de se tratar de relevantes fatores de morbimortalidade neonatal e infantil que podem ser amenizados com ações de baixo custo, a exemplo do desenvolvimento de educação em saúde, acompanhamento do pré-natal com qualidade, resolutividade diante dos problemas evidenciados, sensibilização dos profissionais para o atendimento humanizado e mudança no modelo de assistência à essa população.

Acredita-se que este estudo possa contribuir para subsidiar a posteriori, planejamentos de gestão em saúde, bem como para a avaliação de políticas públicas, criação de estratégias e ações direcionadas para a promoção e proteção à saúde materno-infantil no Amazonas, fortalecer e direcionar o cuidado e atenção para melhoria da assistência na promoção e proteção à saúde dessa população. Como limitações aponta-se a falta de registros e/ou inadequação destes na caderneta da gestante, bem como nos prontuários. 


\section{REFERÊNCIAS}

1. ARNOLD L, et al. Low birthweight increases riskfor cardiovascular disease hospitalisations in a remote Indigenous Australian community - a prospective cohortstudy. Aust N Z J Public Health. 2016; 40 (Suppl 1): S102-S6.

2. BARRETO CTG, et al. Fatores associados ao Baixo Peso ao Nascer em Populações Indígenas: Uma revisão sistemática da literatura mundial. Rev. Bras. Saúde Mater. Infant., Recife, 2019; 19(1): 25-26 41.

3. BRAGA IF, et al. Percepções de adolescentes sobre o apoio social na maternidade no contexto da atenção primária. Universidade de São Paulo. Ribeirão Preto - SP, Brasil. Esc Anna Nery 2014; 18(3): 448-455.

4. BRASIL. Ministério da Saúde. Secretaria de Atenção à Saúde. Departamento de Ações Programáticas Estratégicas. Gestação de alto risco: manual técnico / Ministério da Saúde, Secretaria de Atenção à Saúde, Departamento de Ações Programáticas Estratégicas. - 5. ed. - Brasília: Editora do Ministério da Saúde, 2012.

5. BRASIL. Ministério da Saúde. Secretaria de Atenção à Saúde. Departamento de Ações Programáticas Estratégicas. Atenção humanizada ao recém-nascido de baixo peso : Método Canguru : manual técnico / Ministério da Saúde, Secretaria de Atenção à Saúde, Departamento de Ações Programáticas Estratégicas. - 2. ed., 1. reimpr. - Brasília : Editora do Ministério da Saúde, 2013.

6. CHERMONT A, et al. Fatores associados ao baixo peso ao nascer em uma maternidade pública. Para Res Med J. 2019; 3(1): e03.

7. FIGUEIRÓ-FILHO EA, et al. Variáveis perinatais e associação de recém-nascidos de muito baixo peso ao nascer em hospital público universitário do Brasil. Rev Bras Ginecol Obstet. 2014; 36(1): 10-6.

8. GOIS ALC, et al. Infecção trato urinário e trabalho de parto prematuro: a realidade em uma maternidade referência para alto risco em Aracaju (SE). Caderno de graduação-ciências biológicas e da saúde, 2010; 11(11).

9. GONZAGA ICA, et al. Atenção pré-natal e fatores de risco associados à prematuridade e baixo peso ao nascer em capital do nordeste brasileiro. Ciência \& Saúde Coletiva, 2016; 21(6): 1965-1974.

10. GUIMARÃES EAA, et al. Prevalência e fatores associados à prematuridade em Divinópolis, Minas Gerais, 2008-2011: análise do Sistema de Informações sobre Nascidos Vivos Epidemiol. Serv. Saude, 2017; 26(1): 91-98.

11. MELO EC, et al. Fatores associados à qualidade do pré-natal: uma abordagem ao nascimento prematuro. Rev Esc Enferm USP, 2015; 49(4): 540-549.

12. MOREIRA AI, et al. Baixo peso ao nascer e seus fatores associados. Einstein (São Paulo). 2018;16(4):eAO4251.

13. OLIVEIRA AA, et al. Fatores associados ao nascimento pré-termo: da regressão logística à modelagem com equações estruturais. Cad. Saúde Pública 2019; 35(1): e00211917.

14. SILVA LB, CAPAZ R. Preceptoria: uma Interface entre Educação e Saúde no SUS. In: SILVA LB, RAMOS A, organizadoras. Serviço Social, saúde e questões contemporâneas: reflexões críticas sobre a prática profissional. São Paulo: Papel Social; 2013; 201-215.

15. SILVA PNS, et al. Associação entre doença periodontal, parto prematuro e baixo peso ao nascer. Revista Cubana de Estomatología 2018; 55(1): 26-33.

16. SILVA CA, DALBELLO-ARAUJO M. Programa de Residência Multiprofissional em Saúde: o que mostram as publicações, SAÚDE DEBATE | RIO DE JANEIRO, 2019; 43(123).

17. VIELLAS EF, et al. Gravidez recorrente na adolescência e os desfechos negativos no recém- nascido. Rev Bras Epidemiol 2012; 15(3): 443-54.

18. TEIXEIRA AG, et al. Perfil de mães e o desfecho do nascimento prematuro ou a termo, Cogitare Enferm., 2018; (23)1: e51409.

19. WORLD HEALTH ORGANIZATION (WHO). Global nutrition targets 2025: low birth weight policy brief [Internet]. Geneva: WHO; 2014.

20. WORLD HEALTH ORGANIZATION (WHO); Recomendações da OMS sobre cuidados pré-natais para uma experiência positiva na gravidez. Organização Mundial da Saúde 2016. 